\title{
Pausa activa y clima laboral en áreas académicas y administrativas de la Universidad Peruana Unión, 2018
}

\section{Active break and work climate in academic and administrative areas of Universidad Peruana Unión, 2018}

\author{
Agata Samantha Ibañez Asto ${ }^{1}$
}

\begin{abstract}
RESUMEN
Objetivo: Determinar la relación entre el nivel de práctica de Pausa Activa y Clima Laboral en áreas académicas y administrativas de la Universidad Peruana Unión. Metodología: El diseño de estudio fue cuantitativo no probabilístico, tipo descriptivo, correlacional de corte transversal; se aplicó la encuesta de pausa activa y el clima laboral a 200 personas que participaron anteriormente del programa "Pausa activa en la Universidad Peruana Unión". Resultados: El 59.5\% a veces practica la pausa activa y el $86 \%$ mantiene un clima laboral bueno, en el análisis estadístico de correlación Tau-b de Kendall tuvo un puntaje $p$-valor $=0,307$, lo que indica que no existe relación entre pausa activa y el clima laboral, al igual que las dimensiones de Organización p-valor $=0,178$, Comunicación $p$-valor $=0,525$, Condiciones de trabajo $p$-valor $=0,376$ que tampoco tienen relación significativa, a diferencia de las dimensiones de Interacción social $p$-valor $=0,038$ y el de Identidad y pertenencia $p$-valor $=0,025$, que tienen relación significativa con la variable de pausa activa. Conclusiones: No existe relación en ambas variables, ni en tres dimensiones (organización, comunicación y condiciones de trabajo), solo en dos de ellas (interacción social e identidad y pertenencia).
\end{abstract}

Palabras claves: Pausa activa, Salud ocupacional, Clima laboral, Clima organizacional.

\begin{abstract}
Objective: To determine the relationship between the level of practice of Active Pause and Labor Climate in academic and administrative areas of the Universidad Peruana Unión. Methodology: The study design was non-probabilistic quantitative, descriptive type, cross-sectional correlation; The active pause survey and the work climate were applied to 200 people who previously participated in the "Active Pause at Universidad Peruana Unión" program "Active Pause at the Universidad Peruana Unión". Results: The $59.5 \%$ sometimes practice the active pause and $86 \%$ maintain a good working climate, in the statistical analysis of correlation Tau-b of Kendall had a p-value score $=0.307$, which indicates that there is no relationship between active pause and work climate, as well as the dimensions of Organization $p$-value $=0.178$, Communication $p$-value $=0.525$, Working conditions $p$-value $=0.376$ which also have no significant relationship, unlike the dimensions of Social interaction $p$-value $=0.038$ and that of Identity and belonging $p$-value $=0.025$, which have a significant relationship with the active pause variable. Conclusion: It was not found a relationship in both variables, nor in three dimensions (organization, communication and working conditions), only in two of them (social interaction and identity and belonging). statistically significant correlation between CVR and lifestyle $(p=0.234)$, nevertheless it was found a correlation between CVR and eating habits $(p=0.012)$.
\end{abstract}

Keywords: Active pause, Occupational health, Work climate, Organizational

${ }^{1}$ Enfermera, Servicio de Medicina, Clínica Good Hope, Lima, Perú 


\section{INTRODUCCIÓN}

La Organización Mundial de la Salud (OMS, 2014) refiere que muchos de los problemas laborales en el trabajo de oficina ocasionan desgaste del trabajador, aumento considerable de inactividad física además de estrés. Sin lugar a dudas, estamos frente a uno de los graves problemas del siglo XXI y que provoca la muerte de más de 35 millones de personas a nivel mundial.

Corcuera (2016), señala que gran parte de los síntomas que se presentan en el sistema músculo esquelético y que producen malestares en algunas áreas del cuerpo, impiden ciertos movimientos que pueden afectar al desarrollo normal de la actividad laboral cotidiana. Según una investigación realizada por la Organización Internacional del Trabajo (OIT, 2013), el número de trabajadores con enfermedades profesionales asciende a los 35 millones anualmente. De dichas enfermedades, el $35 \%$ se manifiesta como crónica, el $10 \%$ desarrolla incapacidad continua y el $1 \%$ es causante de muerte. En Venezuela, el porcentaje de las enfermedades profesionales relacionado a trastornos músculo esqueléticos se eleva a $76 \%$; mientras que las de tipo mental y de comportamiento comprende un $14 \%$; finalmente, las asociadas a afecciones pulmonares, un $13 \%$. Las cifras no son diferentes en Colombia, donde el $80.8 \%$ de las enfermedades profesionales corresponde a síntomas músculo-esqueléticos.

Sobre la base de estos datos alarmantes, Vallejo (2013) señala que se han tomado medidas para promover estilos de vida saludables. La actividad física, el ejercicio y el deporte son elementos preventivos que permitirán cooperar, en unión con otros hábitos de vida sana, en el desarrollo de una mejor sociedad, más sana, fuerte y saludable, según refiere Gutiérrez (2012).

Se ha encontrado que el programa Pausa Activa no solo contribuye a disminuir el agotamiento y los dolores osteomusculares; sino también el estrés que se genera durante el trabajo diario, se ve disminuido significativamente. Por lo tanto, el programa Pausa activa debe constituirse en una política permanente de toda empresa que esté preocupada por la salud de su personal. De no aplicarse el Programa de Pausas Activas en la Universidad Peruana Unión, la calidad de vida de los trabajadores, no será la adecuada, evidentemente se producirá un estrés laboral por la preocupación de las tareas asignadas, también cambios de humor y agotamiento. Estos problemas que ya forman parte de la dinámica constante dentro de la institución, los alejan del objetivo y meta trazados.

El objetivo fue determinar la relación que existe entre el nivel de práctica de pausa activa y el clima laboral en áreas académicas y administrativas de la Universidad Peruana Unión, 2018.

\section{METODOLOGÍA}

El presente estudio tiene un enfoque cuantitativo de diseño no probabilístico, tipo descriptivo, ya que se describió y correlacionó los elementos que conforman el modelo aquí propuesto, al igual que las características presentes entre la práctica de pausa activa y el clima laboral como medios fundamentales del cambio dentro de la institución; además fue de corte transversal, porque se llevó a cabo en un solo momento, según refiere Hernández Sampieri \& cols. (2010).

La población de estudio estuvo conformada por 800 personas que laboran en la Universidad Peruana Unión como personal docente y administrativo.

La muestra de estudio fue no probabilístico censal, considerando las características de inclusión y exclusión en las áreas de Rectorado, Área financiera, Secretaria general, Posgrado, CRAI, Digeti, Infraestructura, Admisión, Imprenta, Facultad de Ciencias Empresariales, Facultad de Educación, Facultad de Teología, Facultad de Ingeniería y Productos Unión, haciendo un total de 200 personas que conforman la muestra de estudio.

Para medir las variables se utilizó el cuestionario de Percepción del Clima Laboral (CPCL), el cual fue adaptado del trabajo de investigación titulado "Medición del clima laboral requerido para asegurar la efectividad del sistema de gestión de calidad" por Alcántar, Maldonado y Arcos (2012). Este cuestionario está estructurado de la siguiente manera: Modulo I: Indicaciones generales, Módulo II: El cuestionario se constituye de 27 ítems. Su objetivo es conocer el grado en que el encuestado percibe las estructuras y los procesos que se desarrollan en el medio laboral. Además, presenta 5 alternativas en escala de Likert: Totalmente en desacuerdo, En desacuerdo, Indeciso, En acuerdo y Totalmente en acuerdo. El otro instrumento de 
Pausa Activa fue preparado con las preguntas adaptadas del Cuestionario Internacional de actividad física (IPAQ), con propósitos de monitoreo e investigación sobre la práctica y frecuencia de Pausa Activa en las actividades laborales, por lo cual fue validado y llevado a juicio por 5 expertos; El cuestionario comprende 20 preguntas con 3 ítems de opción para marcar en escala de Likert que comprenden: Nunca, A veces, Siempre. Donde Nunca $=1$, A veces $=2$ y Siempre $=3$.

\section{RESULTADOS}

En la tabla 1 se observa que el $42 \%$ del personal que labora en la UPeU, tiene entre 20 a 30 años de edad; asimismo, el $56 \%$ es de sexo masculino. También el $67 \%$ es del área administrativa; en cuanto al tiempo que trabajan en la UpeU, el 56.5\% tiene entre 2 y 10 años. Asimismo, según el tiempo de trabajo en el puesto actual, el $59.5 \%$ tiene menos de 2 años; finalmente, respecto a su condición laboral, el $54 \%$ es nombrado.

Tabla 1

Distribución de las variables sociodemográficas del personal de áreas académicas y administrativas de la Universidad Peruana Unión, 2018.

\begin{tabular}{|c|c|c|}
\hline Datos sociodemográficos & $\mathbf{N}$ & $\%$ \\
\hline \multicolumn{3}{|l|}{ Edad } \\
\hline 20 a 35 & 84 & 42.0 \\
\hline 35 a 50 & 82 & 41.0 \\
\hline 50 a mas & 34 & 17.0 \\
\hline \multicolumn{3}{|l|}{ Sexo } \\
\hline Femenino & 88 & 44.0 \\
\hline Masculino & 112 & 56.0 \\
\hline \multicolumn{3}{|l|}{ Área de trabajo } \\
\hline Académica & 66 & 33.0 \\
\hline Administrativa & 134 & 67.0 \\
\hline \multicolumn{3}{|l|}{ Tiempo de trabajo en la UPeU } \\
\hline Menos de 2 años & 42 & 21.0 \\
\hline Entre 2 y 10 años & 113 & 56.5 \\
\hline Entre 11 y 20 años & 33 & 16.5 \\
\hline Entre 21 y 25 años & 7 & 3.5 \\
\hline Más de 25 años & 5 & 2.5 \\
\hline \multicolumn{3}{|c|}{ Tiempo de trabajo en el puesto actual } \\
\hline Menos de 2 años & 119 & 59.5 \\
\hline Entre 3 y 10 años & 73 & 36.5 \\
\hline Entre 11 y 20 años & 6 & 3.0 \\
\hline Más de 25 años & 2 & 1.0 \\
\hline \multicolumn{3}{|l|}{ Condición laboral } \\
\hline Nombrado & 108 & 54.0 \\
\hline Contratado & 92 & 46.0 \\
\hline Total & 200 & 100.0 \\
\hline
\end{tabular}

En la tabla 2 se observa que del $100 \%$ del personal, el $59.5 \%$ practica a veces la pausa activa, caracterizándose por presentar una cantidad promedio de conductas promotoras de salud. El $20.5 \%$ del personal, practica siempre la pausa activa, es decir, una cantidad elevada de conductas promotoras 
de la salud. Finalmente, el $20 \%$ del personal que nunca practica pausa activa, se caracteriza por presentar una cantidad baja de conductas promotoras de la salud.

Tabla 2

Niveles de práctica de pausa activa en áreas académicas y administrativas de la Universidad Peruana Unión, 2018

\begin{tabular}{lll} 
Pausa activa & $\mathrm{n}$ & $\%$ \\
Nunca & 40 & 20.0 \\
A veces & 119 & 59.5 \\
Siempre & 41 & 20.5 \\
Total & 200 & 100.0 \\
\hline
\end{tabular}

En la tabla 3 se observa que del $100 \%$ del personal, el $86 \%$ evidencia un clima laboral bueno y el 14\%, un clima laboral regular.

Tabla 3

Niveles del clima laboral en áreas académicas y administrativas de la Universidad Peruana Unión, 2018.

\begin{tabular}{lll} 
Clima laboral & $\mathrm{n}$ & $\%$ \\
Regular & 28 & 14.0 \\
Bueno & 172 & 86.0 \\
Total & 200 & 100.0 \\
\hline
\end{tabular}

En la tabla 4 se encuentra que del $100 \%$ del personal, el $59.5 \%$ que practica a veces la pausa activa muestra un clima laboral bueno. Asimismo, el $20.5 \%$ que siempre practica la pausa activa evidencia un nivel de clima laboral bueno. Contradictoriamente, un $20 \%$ que nunca practica la pausa activa presenta un nivel de clima laboral bueno. Para determinar la relación entre ambas variables se empleó la prueba estadística Tau-b de Kendall, en la que se obtuvo un p-valor de 0,307 y un coeficiente de correlación de 0,068 , lo que indica que no existe relación entre pausa activa y clima laboral del personal de la UPeU.

Tabla 4

Coeficiente de correlación entre pausa activa y clima laboral en áreas académicas y administrativas de la Universidad Peruana Unión, 2018.

\begin{tabular}{lllll} 
Pausa cautiva & & Clima Laboral & & \\
Nunca & $\mathrm{N}$ & 7 & Bueno & Total \\
& $\%$ & 3.5 & 33 & 40 \\
A veces & $\mathrm{N}$ & 17 & 16.5 & 20 \\
& $\%$ & 8.5 & 102 & 119 \\
Siempre & $\mathrm{N}$ & 4 & 51 & 59.5 \\
& $\%$ & 2 & 37 & 41 \\
Total & $\mathrm{n}$ & 28 & 18.5 & 20.5 \\
& $\%$ & 14.0 & 172 & 200 \\
& & & 86 & 100 \\
\hline
\end{tabular}

Tau-b de Kendall $=0.087 ; p=0.178$ 


\section{DISCUSIÓN}

Los resultados indicaron que el $59.5 \%$ de los trabajadores a veces practica pausa activa, mientras que el $20.5 \%$ lo hace siempre y solo el $20 \%$ no lo practica (ver tabla 2). De manera contraria el estudio de Díaz, Mena y Rebolledo (2013), realizado en Chile, nos muestra en los resultados un aumento significativo en el nivel de AF ( $t=-1.391)$ con un $95 \%$ de nivel de confianza. La población, que inicialmente clasificó en nivel de AF leve $68.9 \%$, baja a un $59 \%$, los moderados pasan de un $18.3 \%$ a un $16.9 \%$, y los clasificados con $\mathrm{AF}$ intensa que son un $17,8 \%$ aumentaron a un $24 \%$. Fue altamente valorado el programa de pausas activas obteniendo más de un $90 \%$ de aprobación por parte de los funcionarios. También Bernúi (2013), en su estudio, "Programa Pausa Activa para fortalecimiento del personal no docente de la Universidad Peruana Unión, año 2012", indicó la eficacia del programa, los resultados que presentaron, al aplicar el postest, los valores hallados de la $t$ de Student fueron significativos $(p<0,05)$, es decir, el programa Pausa Activa disminuyó el agotamiento y dolores osteomusculares en los 3 meses que duró.

Finalmente, se ha podido observar que el programa Pausa Activa, contribuye a disminuir significativamente tanto el agotamiento y los dolores osteomusculares como el estrés que se genera naturalmente por el trabajo diario.

En cuanto a la variable Clima laboral, en el estudio realizado por Vélez en el Ecuador (2011), se menciona que el ambiente de trabajo que no cumple con los requerimientos necesarios para cuidar este activo, en lugar de motivar lo que se logra es incrementar el deterioro de la salud y la consecuente aparición de enfermedades. Los malos entendidos entre compañeros, por ejemplo, no solo afectan el ambiente laboral, sino que pueden tener repercusiones a nivel familiar y social. En los resultados se encontró el $86 \%$ con un nivel de clima laboral bueno y el $14 \%$ con un nivel de clima laboral regular (ver tabla 3 ). Esto coincide con la investigación de Chávez y Ríos "Clima organizacional y satisfacción laboral de las enfermeras en el servicio de cirugía Hospital Víctor Lazarte Echegaray- Trujillo, 2015 " en la que el $41 \%$ muestra un nivel alto y el $59 \%$ un nivel medio en clima organizacional. Estos resultados difieren con la investigación de Villamil y Sánchez "Influencia del clima organizacional en la satisfacción laboral de los empleados de la municipalidad de Choloma-
Honduras" (2012), que asevera que el 61\% de los empleados, calificaba al clima organizacional de manera desfavorable.

En el estudio se encontró que no existe relación entre las variables Pausa activa y Clima laboral en el personal de áreas académicas y administrativas de la Universidad Peruana Unión, pues se presentó un p-valor $>0,05$. En contraste con el estudio de Ortiz (2016) realizado en Ecuador, en el que los resultados que arrojó el chi cuadrado con un nivel de significancia del 0,05 y un margen de error de 0,95, corrobora que la Pausas Activas presentan un vínculo con el Ambiente de trabajo. La aplicación y evaluación constante del programa Pausa activa, que es implantado como una política permanente dentro de esta institución pública, evidenció resultados favorables como medida de prevención en enfermedades profesionales y en cuanto a su influencia con el clima laboral.

Seguidamente, se encontró que no existe relación entre la variable Pausa activa y la dimensión Organización, que presentó un $p$-valor $>0,05$. Al referirse a la organización, Palma (2004) menciona que es una medida de gestión en la consecución de los objetivos y metas, no alcanzarlas implica ineficacia. Alcantar et al. (2012), señala que es el grado en que el colaborador percibe que existen en la organización procesos y parámetros de acción claros y confiables. Como es obvio, esta dimensión engloba las reglas, procedimientos y niveles jerárquicos dentro de una organización.

Otro resultado señala que no existe relación entre la variable Pausa activa y la dimensión Comunicación, pues presentó un p-valor de $>0,05$. Nuestros resultados difieren de los estudios de Domínguez, Ramírez y García (2013) en los que la dimensión comunicación y el compromiso afectivo tuvieron una relación de 0,582 y en lo que se refiere a la comunicación con el compromiso normativo de 0,319.

Asimismo, los resultados de Uchuypoma (2015) revelan que la comunicación se relaciona significativamente con el compromiso laboral de los trabajadores de la municipalidad de Los Olivos. El valor obtenido fue de $p$-valor $=0,000$ que es menor que el nivel de confianza $\alpha=0,01$, concluyéndose que existe relación significativa entre la comunicación y el compromiso laboral, siendo esta correlación de nivel bajo $(r=, 372)$. 
Tampoco se encontró que no existe relación entre la variable Pausa activa y la dimensión Condiciones de trabajo con un p-valor >0,05. Distinto resultado obtuvo el estudio de Uchuypoma (2015), en el cual la dimensión Condición laboral se relaciona con el Compromiso laboral de los trabajadores de la municipalidad de Los Olivos; los resultados de correlación son de $p$-valor $=0,000$ menor que el nivel de confianza $\alpha=0,01$, concluyéndose que existe relación positiva significativa entre la condición laboral y el compromiso laboral, siendo esta correlación de nivel moderado $(r=$ ,460). Asimismo, Pérez y Rivera (2015), aluden al reconocimiento que los empleados tienen sobre la organización, es decir, aceptan o no que dicha entidad brinda los elementos materiales, económicos y/o psicosociales requeridos para el cumplimiento de las tareas y funciones delegadas.

En cuanto a la variable Pausa activa y la dimensión Interacción social, se encontró que existe una correlación con un p-valor de 0,038 y un coeficiente de correlación de 0,140 . Lo anterior coincide con el estudio de Castillo y Ruiz (2017), que presenta la relación entre el clima organizacional y las relaciones sociales de los colaboradores de la Empresa Municipal de Servicios Eléctricos Utcubamba S.A.C. Mediante el análisis estadístico Rho de Spearman, se obtuvo el coeficiente de 0,557 y un $p$ valor igual a 0,000 ( $p$ valor $<0.05$ ), lo que evidencia que el clima organizacional se relaciona favorablemente con las relaciones sociales de los colaboradores.

Por último, se encontró que existe correlación entre la variable Pausa activa y la dimensión
Identidad y pertenencia, con un p-valor de 0,025 y un coeficiente de correlación de 0,150. Esto coincide con el estudio de Uchuypoma (2015), que concluye que la dimensión de involucramiento laboral se relaciona con el compromiso laboral de los trabajadores de la municipalidad de Los Olivos. Se llegó a determinar que el valor $p=0,000$ es menor que el nivel de confianza $\alpha=0,01$; se concluye, entonces, que existe relación significativa entre Involucramiento laboral y el compromiso laboral, siendo esta correlación moderada $(r=, 477)$.

Por lo tanto, se considera de gran importancia que todas las empresas e instituciones, cualquiera que sea su tamaño o actividad, deben contar con un programa de salud ocupacional destinados a la satisfacción de los trabajadores y el bienestar de los mismos, no solo para proteger la seguridad y salud de los trabajadores sino porque es un deber moral y un mecanismo para potenciar a cada uno de los integrantes de dicha institución mejorando su estado de alerta para responder mejor a los riesgos que existen en su trabajo.

\section{Declaración de financiamiento y de conflictos de interés:}

El estudio fue financiado por los autores, quienes declaran no tener conflictos de interés.

\section{Correspondencia}

Agata Samantha Ibañez Asto

Correo electrónico:

agata_26@upeu.edu.pe 


\section{REFERENCIAS BIBLIOGRÁFICAS}

Alcántar, V., Maldonado, S. y Arcos, J. (2012). Medición del clima laboral requerido para asegurar la efectividad del sistema de gestión de calidad. Revista internacional administración \& finanzas, 5(3), 55-68. Recuperado de ftp:// ftp.repec.org/opt/ReDIF/RePEc/ibf/riafin/riafv5n3-2012/RIAF-V5N3-2012-4.pdf

Bernui Contreras, M. (2013). Programa Pausa Activa para fortalecimiento del personal no docente de la Universidad Peruana Unión, año 2012. Estrategias para el cumplimiento de la misión, 10(2), 8 - 25. Recuperado de file:///D:/Users/ Usuario/Downloads/443-535-1-PB.pdf

Corcuera Maradiegue, M. (2016). Eficacia de la implementación de un programa de Pausas Activas en la disminución de síntomas músculoesqueléticos en trabajadores administrativos de una empresa de servicios de saneamiento. (Tesis de grado, Universidad Cesar Vallejo, Trujillo, Perú). Recuperado de http://repositorio. ucv.edu.pe/bitstream/handle/UCV/563/corcuera_ $\mathrm{mm}$.pdf?sequence $=1$ \&isAllowed $=\mathrm{y}$

Díaz, X., Mena, C. y Rebolledo A. (2012). Propuesta de un programa de promoción de la salud con actividad física en funcionarios públicos. Praxis Educativa, 15(15), 104-109. Recuperado de http://www.redalyc.org/pdf/1531/153122414011. pdf

Hernández Sampieri, R., Fernández Collado, C., Baptista Lucio, M. (2010). Metodología de la investigación. (5 $5^{\mathrm{a}}$ ed.). Mc Graw - Hill / Interamericana Editores, S.A. de C.V. México. Recuperado de: http://www.who.int/features/qa/ health-promotion/es/
Organización Mundial de la Salud. Actividad física. (2014). Recuperado de http://www.who.int/ mediacentre/factsheets/fs385/es/

OIT. (2013). La OIT en América Latina y el Caribe, Avances y perspectivas. Informe Preparado por la Oficina Regional de la OIT por América Latina y el Caribe. Recuperado de http://www.ilo.org/ global/lang--es/index.htm

Ortiz Medrano, M. (2016). Las Pausas Activas y el Clima Laboral en los talleres del gobierno autónomo descentralizado municipal de Pastaza. (Tesis de grado, Universidad Técnica de Ambato, Ecuador). Recuperado de http://repositorio. uta.edu.ec/bitstream/123456789/24118/1/ Mar\%C3\%ADa\%20Alexandra\%200rtiz\%20 Medrano.pdf

Vallejo Rojas, J. (2013). Evaluación de los niveles de actividad física en el personal de oficina del edificio administrativo de la PUCE, para la elaboración de una propuesta de pausa laboral activa como estrategia de atención primaria en salud durante el periodo febrero - abril 2013. (Tesis de grado, Pontificia Universidad Católica del Ecuador, Quito, Ecuador). Recuperado de http://repositorio. puce.edu.ec/bitstream/handle/22000/5988/TPUCE-6256. pdf? sequence=1\&isAllowed=y

Villamil Pineda, O. y Sánchez Fonseca, W. (2012), Influencia del clima organizacional en la satisfacción laboral de los empleados de la Municipalidad de Choloma. Innovare, 1(1), 94112. Recuperado de https://innovare.unitec.edu/ wp-content/uploads/2013/05/Vol1-No5.pdf

Recibido: $16 / 12 / 18$

Aceptado: 10/02/19 